\title{
A Standard Kinetic Energy Reservoir in Gamma-Ray Burst Afterglows
}

\author{
E. Berger ${ }^{1}$ and S. R. Kulkarni ${ }^{1}$ \\ Division of Physics, Mathematics, and Astronomy, California Institute of Technology \\ 105-24, Pasadena, CA 91125 \\ ejb@astro.caltech.edu; srk@astro.caltech.edu \\ D. A. Frail ${ }^{2}$ \\ National Radio Astronomy Observatory, Socorro, NM 87801 \\ dfrail@nrao.edu
}

\begin{abstract}
We present a comprehensive sample of X-ray observations of $41 \gamma$-ray burst (GRB) afterglows, as well as jet opening angles, $\theta_{j}$ for a subset with measured jet breaks. We show that there is a significant dispersion in the X-ray fluxes, and hence isotropic X-ray luminosities $\left(L_{X \text {,iso }}\right)$, normalized to $t=10 \mathrm{hr}$. However, there is a strong correlation between $L_{X \text {,iso }}$ and the beaming fractions, $f_{b} \equiv\left[1-\cos \left(\theta_{j}\right)\right]$. As a result, the true X-ray luminosity of GRB afterglows, $L_{X}=f_{b} L_{X \text {,iso }}$, is approximately constant, with a dispersion of only a factor of two. Since $\epsilon_{e} E_{b} \propto L_{X}$, the strong clustering of $L_{X}$ directly implies that the adiabatic blastwave kinetic energy in the afterglow phase, $E_{b}$, is tightly clustered. The narrow distribution of $L_{X}$ also suggests that $p \approx 2$, that inverse Compton emission does not in general dominate the observed X-ray luminosity, and that radiative losses at $t<10 \mathrm{hr}$ are relatively small. Thus, despite the large diversity in the observed properties of GRBs and their afterglows the energy imparted by the GRB central engine to the relativistic ejecta is approximately constant.
\end{abstract}

Subject headings: gamma rays:bursts - ISM:jets and outflows - shock waves

\section{Introduction}

Gamma-ray bursts (GRBs) exhibit a remarkable diversity: Fluences range from $10^{-7}$ to $10^{-3} \mathrm{erg} \mathrm{cm}^{-2}$, peak energies range from $50 \mathrm{keV}$ to an $\mathrm{MeV}$, and possibly from the Xray to the $\mathrm{GeV}$ band (Fishman \& Meegan 1995), and durations extend from about 2 to 
$10^{3} \mathrm{~s}$ (for the long-duration GRBs). This diversity presumably reflects a dispersion in the progenitors and central engine properties. Perhaps the most impressive feature of GRBs are their brilliant luminosities and isotropic energy releases approaching the rest mass of a neutron star, $E_{\gamma, \text { iso }} \sim 10^{54}$ erg (Kulkarni et al. 1999; Andersen et al. 2000).

The quantity of energy imparted to the relativistic ejecta, $E_{\text {rel }}$, and the quality parameterized by the bulk Lorentz factor, $\Gamma$, are the two fundamental properties of GRB explosions. In particular, extremely high energies push the boundaries of current progenitor and engine models, while low energies could point to a population of sources that is intermediate between GRBs and core-collapse supernovae.

The true energy release depends sensitively on the geometry of the ejecta. If GRB explosions are conical (as opposed to spherical) then the true energy release is significantly below that inferred by assuming isotropy. Starting with GRB 970508 (Waxman, Kulkarni \& Frail 1998; Rhoads 1999) there has been growing observational evidence for collimated outflows, coming mainly from achromatic breaks in the afterglow lightcurves.

In the conventional interpretation, the epoch at which the afterglow lightcurves steepen ("break") corresponds to the time at which $\Gamma$ decreases below $\theta_{j}^{-1}$, the inverse opening angle of the collimated outflow or "jet" (Rhoads 1999). The break happens for two reasons: an edge effect, and lateral spreading of the jet which results in a significant increase of the swept up mass. Many afterglows have $t_{j} \sim 1-$ few days, which are best measured from optical/near-IR lightcurves (e.g. Harrison et al. 1999; Kulkarni et al. 1999; Stanek et al. 1999), while wider opening angles are easily measured from radio lightcurves (e.g. Waxman, Kulkarni \& Frail 1998; Berger et al. 2001).

Recently, Frail et al. (2001) inferred $\theta_{j}$ for fifteen GRB afterglows from measurements of $t_{j}$ and found the surprising result that $E_{\gamma, \text { iso }}$ is strongly correlated with the beaming factor, $f_{b}^{-1}$; here, $f_{b} \equiv\left[1-\cos \left(\theta_{j}\right)\right]$ is the beaming fraction and $E_{\gamma, \text { iso }}$ is the $\gamma$-ray energy release inferred by assuming isotropy. In effect, the true $\gamma$-ray energy release, $E_{\gamma}=f_{b} E_{\gamma, \text { iso }}$ is approximately the same for all the GRBs in their sample, with a value of about $5 \times 10^{50}$ erg (assuming a constant circumburst density, $n_{0}=0.1 \mathrm{~cm}^{-3}$ ). In the same vein, broad-band modeling of several GRB afterglows indicates that the typical blastwave kinetic energy in the adiabatic afterglow phase is $E_{b} \sim 5 \times 10^{50} \mathrm{erg}$, with a spread of about 1.5 orders of magnitude (Panaitescu \& Kumar 2002). However, the general lack of high quality afterglow data severely limits the application of the broad-band modeling method.

Separately, Kumar (2000) and Freedman \& Waxman (2001) noted that the afterglow flux at frequencies above the synchrotron cooling frequency, $\nu_{c}$, is proportional to $\epsilon_{e} d E_{b} / d \Omega$, where $\epsilon_{e}$ is the fraction of the shock energy carried by electrons and $d E_{b} / d \Omega$ is the energy 
of the blastwave per unit solid angle. The principal attraction is that the flux above $\nu_{c}$ does not depend on the circumburst density, and depends only weakly on the fraction of shock energy in magnetic fields, $\epsilon_{B}$. For reasonable conditions (which have been verified by broad-band afterglow modeling, e.g. Panaitescu \& Kumar 2002), the X-ray band (2-10 keV) lies above $\nu_{c}$ starting a few hours after the burst. Thus, this technique offers a significant observational advantage, namely the X-ray luminosity can be used as a surrogate for the isotropic-equivalent afterglow kinetic energy.

Piran et al. (2001) find that the X-ray flux, estimated at a common epoch $(t=11 \mathrm{hr})$, exhibits a narrow distribution of $\log \left(F_{X}\right), \sigma_{l}\left(F_{X}\right)=0.43_{-0.11}^{+0.12}$; here $\sigma_{l}^{2}(x)$ is the variance of $\log (x)$. Taken at face value, the narrow distribution of $F_{X}$ implies a narrow distribution of $\epsilon_{e} d E_{b} / d \Omega$. This result, if true, is quite surprising since if the result of Frail et al. (2001) is accepted then $d E_{b} / d \Omega$ should show a wide dispersion comparable to that of $f_{b}^{-1}$.

Piran et al. (2001) reconcile the two results by the following argument. The relation between $d E_{b} / d \Omega$ and $E_{b}$ can be restated as $\log \left(d E_{b} / d \Omega\right)=\log \left(E_{b}\right)+\log \left(f_{b}^{-1}\right)$. Thus, $\sigma_{l}^{2}\left(d E_{b} / d \Omega\right)=\sigma_{l}^{2}\left(E_{b}\right)+\sigma_{l}^{2}\left(f_{b}^{-1}\right)$. Since $d E_{b} / d \Omega \propto L_{X \text {,iso }}$ (for a constant $\epsilon_{e}$ ) they express, $\sigma_{l}^{2}\left(E_{b}\right)=\sigma_{l}^{2}\left(L_{X, \text { iso }}\right)-\sigma_{l}^{2}\left(f_{b}\right)$. Given the diversity in $\theta_{j}$ (Frail et al. 2001) and the apparent narrowness in $F_{X}$ (above), it would then follow that $E_{b}$ should be very tightly distributed.

However, the approach of Piran et al. (2001) makes a key assumption, namely that $E_{b}$ and $f_{b}^{-1}$ are uncorrelated. This is certainly true when $E_{b}$ is constant, but the assumption then pre-supposes the answer! In reality, a correlation between $E_{b}$ and $f_{b}$ can either increase or decrease $\sigma_{l}^{2}\left(E_{b}\right)$, and this must be addressed directly. Finally, as appears to be the case (see $\S 2), \sigma_{l}^{2}\left(f_{b}^{-1}\right)$ is dominated by bursts with the smallest opening angles, which results in a distinctly different value than the one used by Piran et al. (2001) based only on the observed $\theta_{j}$ values.

In this Letter, we avoid all these concerns by taking a direct approach: we measure the variance in $E_{b} \propto f_{b} L_{X \text {,iso }}$ rather than bounding it through a statistical relation. We show, with a larger sample, that $L_{X \text {,iso }}$ is not as narrowly distributed as claimed by Piran et al. (2001), and in fact shows a spread similar to that of $E_{\gamma, \text { iso }}$. On the other hand, we find that $L_{X \text {,iso }}$ is strongly correlated with $f_{b}^{-1}$. It is this correlation, and not the claimed clustering of $L_{X \text {,iso }}$, that results in, and provides a physical basis for the strong clustering of $L_{X}$ and hence the blastwave kinetic energy, $E_{b}$. 


\section{X-ray Data}

In Table 1 we provide a comprehensive list of X-ray observations for 41 GRB afterglows, as well as temporal decay indices, $\alpha_{X}\left(F_{\nu} \propto t^{\alpha_{X}}\right)$, when available. In addition, for a subset of the afterglows for which jet breaks have been measured from the radio, optical, and/or X-ray emission, we also include the inferred $\theta_{j}$ (Frail et al. 2001; Panaitescu \& Kumar 2002). We calculate $\theta_{j}$ from $t_{j}$ using the circumburst densities inferred from broad-band modeling, when available, or a fiducial value of $10 \mathrm{~cm}^{-3}$, as indicated by the best-studied afterglows (e.g. Yost et al. 2002). This normalization for $n_{0}$ is different from Frail et al. (2001) who used $n_{0}=0.1 \mathrm{~cm}^{-3}$.

For all but one burst we interpolate the measured $F_{X}$ to a fiducial epoch of $10 \mathrm{hr}$ (hereafter, $F_{X, 10}$ ), using the measured $\alpha_{X}$ when available, and the median of the distribution, $\left\langle\alpha_{X}\right\rangle=-1.33 \pm 0.38$ when a measurement is not available. The single exception is GRB 020405 for which the first measurement was obtained $t \approx 41 \mathrm{hr}$, while the inferred jet break time is about $23 \mathrm{hr}$ (Berger et al. in prep). In this case, we extrapolate to $t=10 \mathrm{hr}$ using $\alpha_{X}=-1.69$ for $t>23 \mathrm{hr}$ and $\alpha_{X}=-0.78$ for $t<23 \mathrm{hr}$. We list the values of $F_{X, 10}$ in Table 2.

In Figure 1 we plot the resulting distribution of $F_{X, 10}$. For comparison we also show the distribution of $\gamma$-ray fluences from the sample presented by Bloom, Frail \& Sari (2001) and updated from the literature. Clearly, while the distribution of X-ray fluxes is narrower than that of the $\gamma$-ray fluences, $\sigma_{l}\left(f_{\gamma}\right)=0.79_{-0.08}^{+0.10}$, it still spans $\sim 2.5$ orders of magnitude, i.e. $\sigma_{l}\left(F_{X, 10}\right)=0.57_{-0.06}^{+0.07}$. The value of $\sigma_{l}\left(F_{X, 10}\right)$, and all variances quoted below, are calculated by summing the Gaussian distribution for each measurement, and then fitting the combined distribution with a Gaussian profile.

We translate the observed X-ray fluxes to isotropic luminosities using:

$$
L_{X, \text { iso }}(t=10 \mathrm{hr})=4 \pi d_{L}^{2} F_{X, 10}(1+z)^{\alpha_{X}-\beta_{X}-1}
$$

We use $\beta_{X} \approx-1.05$, the weighted mean value for X-ray afterglows (De Pasquale et al. 2002), and the median redshift, $\langle z\rangle=1.1$, for bursts that do not have a measured redshift. The resulting distribution of $L_{X \text {,iso }}, \sigma_{l}\left(L_{X \text {,iso }}\right)=0.68_{-0.09}^{+0.17}$, is wider than that of $F_{X}$ due to the dispersion in redshifts. We note that this is significantly wider than the value quoted by Piran et al. $(2001)$ of $\sigma_{l}\left(L_{X \text {,iso }}\right) \approx 0.43$ based on a smaller sample. Using the same method we find $\sigma_{l}\left(E_{\gamma, \text { iso }}\right)=0.92_{-0.08}^{+0.12}$.

In the absence of a strong correlation between $f_{b}$ and $L_{X \text {,iso }}$, the above results indi-

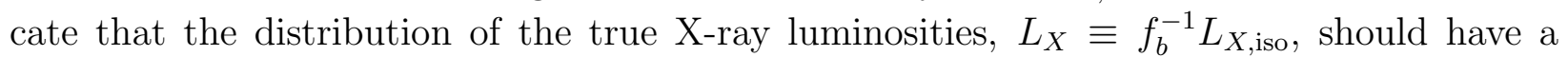
wider dispersion than either $L_{X \text {,iso }}$ or $f_{b}$, for which we find $\sigma_{l}\left(f_{b}\right)=0.52_{-0.12}^{+0.13}$ (Frail et al. 
2001). Instead, when we apply the individual beaming corrections for those bursts that have a measured $\theta_{j}$ and redshift ${ }^{1}$ (see Table 2 ), we find a significantly narrower distribution, $\sigma_{l}\left(L_{X}\right)=0.32_{-0.06}^{+0.10}$.

\section{Beaming Corrections and Kinetic Energies}

The reduced variance of $L_{X}$ compared to that of $L_{X \text {,iso }}$ requires a strong correlation between $L_{X \text {,iso }}$ and $f_{b}^{-1}$, such that bursts with a brighter isotropic X-ray luminosity are also more strongly collimated. Indeed, as can be seen from Figure 2 the data exhibit such a correlation. Ignoring the two bursts which are obvious outliers (980326 and 990705), as well as GRBs 980329 and 980519, which do not have a measured redshift, we find $L_{X \text {,iso }} \propto f_{b}^{0.88}$. The linear correlation coefficient between $L_{X \text {,iso }}$ and $f_{b}$ indicates a probability that the two quantities are not correlated of only $4.6 \times 10^{-4}$. For $E_{\gamma \text {,iso }}$ and $f_{b}$ we find a similar probability of $4.2 \times 10^{-4}$ that the two quantities are not correlated.

Thus, as with the $\gamma$-ray emission, the afterglow emission also exhibits strong luminosity diversity due to strong variations in $f_{b}$. Therefore, the mystery of GRBs is no longer the energy release but understanding what aspect of the central engine drives the wide diversity of $f_{b}$.

We note that there are four possible outliers in the correlation between $L_{X \text {,iso }}$ and $f_{b}^{-1}$. The afterglows of GRBs 980326 and 980519 exhibit rapid fading (Groot et al. 1998; Vrba et al. 2000), which has been interpreted as the signature of an early jet break. However, it is possible that the rapid fading is instead due to a $\rho \propto r^{-2}$ density profile, and in fact for GRB 980519 such a model indicates $\theta_{j} \approx 0.12$, 3 times wider than in the constant density model. This is sufficient to bring GRB 980519 into agreement with the observed correlation. The redshift of GRB 980329 is not known, but with $z=2$ it easily agrees with the correlation. Finally, the X-ray flux and jet opening angle for GRB 990705 are poorly characterized due to contamination from a nearby source (De Pasquale et al. 2002) and a poor optical lightcurve (Masetti et al. 2000).

\section{Discussion and Conclusions}

We have presented a comprehensive compilation of early X-ray observations of 41 GRBs, from which we infer $F_{X, 10}$, the flux in the $2-10 \mathrm{keV}$ band at $10 \mathrm{hr}$. As first pointed by

\footnotetext{
${ }^{1}$ These do not include GRB 990705 which is poorly characterized; see $\S 3$.
} 
Kumar (2000) and Freedman \& Waxman (2001), the afterglow luminosity above the cooling frequency is $L_{X, \text { iso }} \propto \epsilon_{e} E_{b \text {,iso }}$ where $E_{b \text {,iso }}$ is the isotropic-equivalent explosion kinetic energy. More importantly, the flux is independent of the ambient density and weakly dependent on $\epsilon_{B}$. For all well modeled afterglows, the cooling frequency at $10 \mathrm{hr}$ is below the X-ray band. Thus, $F_{X, 10}$ can be utilized to yield information about the kinetic energy of GRBs.

Earlier work (Piran et al. 2001) focussed on statistical studies of $F_{X, 10}$ and found the very surprising result that it is narrowly clustered. By assuming that the true kinetic energy, $E_{b}=E_{b, \text { iso }} f_{b} \propto L_{X}=L_{X \text {,iso }} f_{b}$, and $f_{b}$ (the beaming factor) are uncorrelated, the authors deduced that $L_{X}$ and thus $E_{b}$ are even more strongly clustered. However, this approach is weakened by assuming (in effect) the answer. Furthermore, the approach of Piran et al. (2001) which relies on subtracting variances is very sensitive to measurement errors. To illustrate this point, we note $\sigma_{l}^{2}\left(L_{X \text {,iso }}\right)=0.68_{-0.09}^{+0.17}$ for the entire sample presented here, whereas $\sigma_{l}^{2}\left(f_{b}\right)=0.52_{-0.12}^{+0.13}$. Thus, $\sigma_{l}^{2}\left(L_{X}\right)=0.16_{-0.21}^{+0.30}$ may be negative using the statistical approach.

In contrast to the statistical approach, we take the direct approach and estimate the true kinetic energy, $E_{b} \propto L_{X \text {,iso }} f_{b}$, by using the measured $L_{X \text {,iso }}$ and inferred $f_{b}$. The advantage of our approach is that we do not make assumptions of correlations (or lack thereof) and more importantly we do not subtract variances. We directly compute the variance of the desired physical quantity, namely $L_{X}$, and find that it is strongly clustered.

Even more importantly, with our direct approach we have uncovered the physical reason for the wide dispersion in $L_{X \text {,iso }}$ and the clustering of $L_{X}$, namely the dispersion in jet opening angles.

$L_{X}$ is related to the physical quantities as follows (Freedman \& Waxman 2001):

$$
\epsilon_{e} E_{b} \propto A L_{X} Y^{\epsilon}
$$

where

$$
Y \equiv B \epsilon_{e}^{-3} \epsilon_{B}^{-1} L_{X, \text { iso }}^{-1}
$$

Here $\epsilon \equiv(p-2) /(p-1)$, as well as $A$ and $B$ depend to some extent on the details of the electron distribution (power law versus relativistic Maxwellian; the value of power law index, $p)$.

There is no reason to expect that $L_{X}$ should be clustered. However, one can argue that the microphysics should be the same for each GRB afterglow, in particular $\epsilon_{e}$ and $p$. The best studied afterglows appear to favor $p=2.2$ (e.g. Frail, Waxman \& Kulkarni 2000; Galama et al. 1998), a value also favored by our current theoretical knowledge of shock acceleration (see Ostrowski \& Bednarz 2002 and references therein). In addition, as already indicated by 
the $\gamma$-ray observations, there is evidence supporting strong clustering of explosion energies in GRBs (Frail et al. 2001).

Given these reasonable assumptions, a strong clustering of $L_{X}$ makes sense if the physical quantities that are responsible for $L_{X}$ are clustered. As can be seen from Equation 2, this would require that $L_{X}$ be linearly related to $E_{b}$. Such a relation is possible if three conditions are met.

First, the afterglow X-ray emission on timescales of $10 \mathrm{hr}$ must be primarily dominated by synchrotron emission (which is the basis of Equation 2). Contribution from inverse Compton (IC) emission, which depends strongly on $n_{0}$ and $\epsilon_{B}$ (Sari \& Esin 2001), is apparently not significant. A possible exception is GRB 000926 (Harrison et al. 2001), but even there the IC contribution is similar to that from synchrotron emission.

Second, the energy radiated by the afterglow from the time of the explosion to $t=10 \mathrm{hr}$ cannot be significant. This constrains the radiative losses at early time to at most a factor of few.

Third, $p$ must be relatively constant (as one may expect in any case from insisting that the microphysics should not be different for different bursts). For example, changing $p$ from a value of 1.5 to 3 results in $Y^{\epsilon}$ ranging from 0.003 to 117, a factor of 39,000! Even small changes in $p$, e.g. from $p=1.75$ to $p=2.25$, result in a factor of 8 change in $Y^{\epsilon}$. In contrast, some afterglow models yield values of $p$ significantly below 2 (e.g. Panaitescu \& Kumar 2002), while others have $p$ approaching 3 (Chevalier \& Li 2000). Our results, on the other hand, indicate that one should set $p \approx 2$ and attribute apparent deviant values of $p$ to external environment or energy injection from the central source.

We end with an interesting conclusion from the results presented here. Since both the prompt and afterglow emission exhibit a strong correlation with $f_{b}$, which is determined from late-time observations (hours to weeks after the burst), the resulting constancy of both $E_{\gamma}$ and $E_{b}$, indicates that GRB jets must be relatively homogeneous and maintain a simple conical geometry all the way from internal shocks $\left(\sim 10^{13}-10^{14} \mathrm{~cm}\right)$ to the epoch of jet break $\left(\sim 10^{17} \mathrm{~cm}\right)$. This rules out the idea that brighter bursts are due to bright spots along specific lines of sight (Kumar \& Piran 2000), or that GRB jets have a strong energy and/or Lorentz factor gradient across their surface (Rossi, Lazzati \& Rees 2002). It is indeed remarkable that the simplest description of jets is fully consistent with the observations.

SRK thanks S. Phinney for valuable discussions. We acknowledge support from SNF and NASA grants. 


\section{REFERENCES}

Amati, L. et al. 2000a, GRB Circular Network, 885, 1.

Amati, L. et al. 2000b, Science, 290, 953.

Andersen, M. I. et al. 2000, A\&A, 364, L54.

Antonelli, L. A. et al. 1999, A\&AS, 138, 435.

Antonelli, L. A. et al. 2000, GRB Circular Network, 561, 1.

Berger, E. et al. 2001, ApJ, 556, 556.

Bloom, J. S., Frail, D. A., and Sari, R. 2001, AJ, 121, 2879.

Bloom, J. S. et al. 1999, Nature, 401, 453.

Chevalier, R. A. and Li, Z. 2000, ApJ, 536, 195.

De Pasquale, M. et al. 2002, Submitted to ApJ; astro-ph/0212298.

Feroci, M. et al. 1998, A\&A, 332, L29.

Feroci, M. et al. 2001, A\&A, 378, 441.

Feroci, M. et al. 2000, GRB Circular Network, 685, 1.

Fishman, G. J. and Meegan, C. A. 1995, ARA\&A, 33, 415.

Frail, D. A. et al. 2001, ApJ, 562, L55.

Frail, D. A., Waxman, E., and Kulkarni, S. R. 2000, ApJ, 537, 191.

Freedman, D. L. and Waxman, E. 2001, ApJ, 547, 922.

Frontera, F. et al. 2000, ApJ, 540, 697.

Frontera, F. et al. 1999, GRB Circular Network, 401, 1.

Frontera, F. et al. 1998, ApJ, 493, L67.

Frontera, F. et al. 2001, GRB Circular Network, 950, 1.

Galama, T. J., Wijers, R. A. M. J., Bremer, M., Groot, P. J., Strom, R. G., Kouveliotou, C., and van Paradijs, J. 1998, ApJ, 500, L97+. 
Groot, P. J. et al. 1998, ApJ, 502, L123+.

Harrison, F. A. et al. 1999, ApJ, 523, L121.

Harrison, F. A. et al. 2001, ApJ, 559, 123.

in 't Zand, J. J. M. et al. 1998, ApJ, 505, L119.

in’t Zand, J. J. M. et al. 2001, ApJ, 559, 710.

Jaunsen, A. O. et al. 2001, ApJ, 546, 127.

Kulkarni, S. R. et al. 1999, Nature, 398, 389.

Kumar, P. 2000, ApJ, 538, L125.

Kumar, P. and Piran, T. 2000, ApJ, 535, 152.

Marshall, F. E. and Takeshima, T. 1998, GRB Circular Network, 58, 1.

Marshall, F. E., Takeshima, T., Kippen, T., and Giblin, R. M. 2000, GRB Circular Network, $519,1$.

Masetti, N. et al. 2000, A\&A, 354, 473.

Mirabal, N., Paerels, F., and Halpern, J. P. 2002, ApJ (submitted), astro-ph/0209516.

Murakami, T., Ueda, Y., Ishida, M., Fujimoto, R., Yoshida, A., and Kawai, N. 1997, IAU Circ., 6722, 1.

Nicastro, L. et al. 1999a, A\&AS, 138, 437.

Nicastro, L., Antonelli, L. A., Dadina, M., Daniele, M. R., Costa, E., and Pian, E. 1999b, IAU Circ., 7213, 2.

Ostrowski, M. and Bednarz, J. 2002, A\&A, 394, 1141.

Panaitescu, A. and Kumar, P. 2002, ApJ, 571, 779.

Pian, E. et al. 2001, A\&A, 372, 456.

Piran, T., Kumar, P., Panaitescu, A., and Piro, L. 2001, ApJ, 560, L167.

Piro, L. 2001, in AIP Conf. Proc. 599: X-ray Astronomy: Stellar Endpoints, AGN, and the Diffuse X-ray Background, 295. 
Piro, L. et al. 1998, A\&A, 331, L41.

Piro, L. et al. 2002, ApJ (submitted), astro-ph/0201282.

Price, P. A., Bloom, J. S., Goodrich, R. W., Barth, A. J., Cohen, M. H., and Fox, D. W. 2002, GRB Circular Network, 1475, 1.

Price, P. A. and et al. 2002, ApJ (submitted), astro-ph/0208008.

Reeves, J. N. et al. 2002, Nature, 416, 512.

Rhoads, J. E. 1999, ApJ, 525, 737.

Rossi, E., Lazzati, D., and Rees, M. J. 2002, MNRAS, 332, 945.

Sari, R. and Esin, A. A. 2001, ApJ, 548, 787.

Smith, D. A. et al. 2002, ApJS, 141, 415.

Stanek, K. Z., Garnavich, P. M., Kaluzny, J., Pych, W., and Thompson, I. 1999, ApJ, 522, L39.

Takeshima, T., Markwardt, C., Marshall, F., Giblin, T., and Kippen, R. M. 1999, GRB Circular Network, 478, 1.

Vanderspek, R., Marshall, H. L., Ford, P. G., and Ricker, G. R. 2002, GRB Circular Network, $1504,1$.

Vrba, F. J. et al. 2000, ApJ, 528, 254.

Vreeswijk, P. M. et al. 1999, ApJ, 523, 171.

Watson, D., Reeves, J. N., Osborne, J. P., Tedds, J. A., O'Brien, P. T., Tomas, L., and Ehle, M. 2002, A\&A, 395, L41.

Waxman, E., Kulkarni, S. R., and Frail, D. A. 1998, ApJ, 497, 288.

Yost, S. A. et al. 2002, ApJ, 577, 155. 
Table 1. X-ray Afterglow Data

\begin{tabular}{|c|c|c|c|c|c|c|}
\hline GRB & $z$ & $\begin{array}{l}\text { Epoch } \\
\text { (hrs) }\end{array}$ & $\begin{array}{c}\text { Flux } \\
\left(10^{-13} \mathrm{erg} \mathrm{cm}^{-2} \mathrm{~s}^{-1}\right)\end{array}$ & $\alpha_{X}$ & $\theta_{\text {jet }}$ & Ref. \\
\hline \multirow[t]{2}{*}{970111} & . & 24.0 & $1.05 \pm 0.46$ & $-0.4 \pm 3.2^{a}$ & $\ldots$ & 1,2 \\
\hline & & 30.7 & $0.95 \pm 0.34$ & & $\cdots$ & 2 \\
\hline \multirow[t]{3}{*}{970228} & 0.695 & 8.5 & $33.8 \pm 3.3$ & $-1.27 \pm 0.14$ & $\ldots$ & 2,3 \\
\hline & & 12.7 & $28 \pm 4$ & & & 2 \\
\hline & & 92.4 & $1.5 \pm 0.4$ & & & 2 \\
\hline \multirow[t]{2}{*}{970402} & . & 9.9 & $2.9 \pm 0.4$ & $-1.35 \pm 0.55$ & $\ldots$ & 2 \\
\hline & & 16.8 & $1.5 \pm 0.4$ & & & 2 \\
\hline \multirow[t]{3}{*}{970508} & 0.835 & 13.1 & 7.13 & $-1.1 \pm 0.1$ & 0.391 & 4,5 \\
\hline & & 72.3 & $4.3 \pm 0.5$ & & & 2 \\
\hline & & 104 & $2.3 \pm 0.7$ & & & 2 \\
\hline 970815 & .. & 89.6 & $<1$ & $\ldots$ & $\ldots$ & 6 \\
\hline \multirow[t]{2}{*}{970828} & 0.958 & 4.0 & 118 & -1.42 & 0.128 & 5,7 \\
\hline & & 42.6 & 4.1 & & & 7 \\
\hline \multirow[t]{2}{*}{971214} & 3.418 & 8.1 & $9.0 \pm 0.9$ & $-1 \pm 0.2$ & $>0.100$ & 2,5 \\
\hline & & 28.9 & $2.1 \pm 0.4$ & & & 2 \\
\hline 971227 & $\cdots$ & 16.5 & $2.5 \pm 0.7$ & $-1.12 \pm 0.06$ & $\cdots$ & 8 \\
\hline 980326 & $\sim 1^{b}$ & 8.5 & $<16$ & $\ldots$ & $<0.110$ & 9 \\
\hline \multirow[t]{5}{*}{980329} & $\ldots$ & 8.4 & $14 \pm 2.1$ & $-1.55 \pm 0.3$ & 0.081 & 10,11 \\
\hline & & 11.8 & $6.2 \pm 1.2$ & & & 10 \\
\hline & & 16.4 & $3.4 \pm 1.0$ & & & 10 \\
\hline & & 23.7 & $2.7 \pm 0.7$ & & & 10 \\
\hline & & 43.6 & $1.1 \pm 0.4$ & & & 10 \\
\hline 980515 & $\ldots$ & 11 & $2.0_{-0.9}^{+0.5}$ & $\ldots$ & $\ldots$ & 12 \\
\hline \multirow[t]{4}{*}{980519} & $<2^{c}$ & 10.9 & $5.3 \pm 1.0$ & $-1.7 \pm 0.7$ & 0.040 & 13,14 \\
\hline & & 15.3 & $2.0 \pm 0.4$ & & & 13 \\
\hline & & 21.5 & $1.6 \pm 0.5$ & & & 13 \\
\hline & & 27.2 & $0.8 \pm 0.4$ & & & 13 \\
\hline \multirow[t]{2}{*}{980613} & 1.096 & 9.9 & $7.1 \pm 1.9$ & $-0.92 \pm 0.62$ & $>0.226$ & 2 \\
\hline & & 23.4 & $4.0 \pm 0.8$ & & & 2 \\
\hline 980703 & 0.966 & 34.0 & $4.0 \pm 1$ & $-1.24 \pm 0.18$ & 0.200 & 2,15 \\
\hline
\end{tabular}


Table 1-Continued

\begin{tabular}{|c|c|c|c|c|c|c|}
\hline GRB & $z$ & $\begin{array}{c}\text { Epoch } \\
\text { (hrs) }\end{array}$ & $\begin{array}{c}\text { Flux } \\
\left(10^{-13} \mathrm{erg} \mathrm{cm}^{-2} \mathrm{~s}^{-1}\right)\end{array}$ & $\alpha_{X}$ & $\theta_{\text {jet }}$ & Ref. \\
\hline 981226 & $\ldots$ & 14.0 & 4.0 & $-1.3 \pm 0.4$ & $\ldots$ & 16 \\
\hline \multirow[t]{2}{*}{990123} & 1.600 & 6.4 & $124 \pm 11$ & $-1.41 \pm 0.05$ & 0.089 & 2,5 \\
\hline & & 23.4 & $19.1 \pm 2.2$ & & & 2 \\
\hline 990217 & $\ldots$ & 11 & $<1.1$ & .. & $\ldots$ & 12 \\
\hline \multirow[t]{10}{*}{990510} & 1.619 & 8.7 & $47.8 \pm 3.1$ & $-1.41 \pm 0.18$ & 0.054 & $5,14,17$ \\
\hline & & 10.1 & $40.5 \pm 2.6$ & & & 17 \\
\hline & & 11.7 & $32.8 \pm 3.7$ & & & 17 \\
\hline & & 13.4 & $22.8 \pm 2.8$ & & & 17 \\
\hline & & 15.3 & $24.1 \pm 2.7$ & & & 17 \\
\hline & & 17.1 & $18.5 \pm 3.1$ & & & 17 \\
\hline & & 19.1 & $20.9 \pm 2.3$ & & & 17 \\
\hline & & 24.0 & $12.1 \pm 1.4$ & & & 17 \\
\hline & & 26.3 & $9.9 \pm 1.1$ & & & 17 \\
\hline & & 29.4 & $7.8 \pm 1.1$ & & & 17 \\
\hline 990627 & $\cdots$ & 11.9 & 3.5 & $\ldots$ & $\ldots$ & 18 \\
\hline \multirow[t]{4}{*}{990704} & $\cdots$ & 10.1 & $10.1 \pm 2.9$ & $-1.3 \pm 0.3$ & $\cdots$ & 19 \\
\hline & & 13.4 & $8.9 \pm 2.2$ & & & 19 \\
\hline & & 23.3 & $3.1 \pm 2.0$ & & & 19 \\
\hline & & 26.8 & $2.9 \pm 1.6$ & & & 19 \\
\hline 990705 & 0.840 & 14.5 & $1.9 \pm 0.6$ & $\cdots$ & 0.096 & 5,20 \\
\hline \multirow[t]{2}{*}{990806} & $\ldots$ & 13.6 & $5.5 \pm 1.5$ & $-1.4 \pm 0.7$ & $\ldots$ & 21 \\
\hline & & 34.3 & $1.5 \pm 0.6$ & & & 21 \\
\hline 990907 & $\ldots$ & 11 & $10.2 \pm 5.6$ & $\cdots$ & $\cdots$ & 12 \\
\hline 991014 & $\ldots$ & 11 & $4.0_{-1.2}^{+1.4}$ & $\ldots$ & $\ldots$ & 12 \\
\hline \multirow[t]{2}{*}{991216} & 1.020 & 4.0 & $1240 \pm 40$ & $-1.61 \pm 0.07$ & 0.051 & $5,14,22$ \\
\hline & & 10.9 & $250 \pm 10$ & & & 22 \\
\hline 000115 & $\ldots$ & 2.9 & 270 & $<-1$ & $\ldots$ & 23 \\
\hline 000210 & 0.846 & 11 & $4.0 \pm 1.0$ & $-1.38 \pm 0.03$ & $\cdots$ & 24 \\
\hline \multirow[t]{2}{*}{000214} & $\ldots$ & 14.9 & 5 & -1.8 & $\cdots$ & 25 \\
\hline & & 22.1 & 2.5 & & & 25 \\
\hline
\end{tabular}


Table 1 - Continued

\begin{tabular}{|c|c|c|c|c|c|c|}
\hline GRB & $z$ & $\begin{array}{l}\text { Epoch } \\
(\mathrm{hrs})\end{array}$ & $\begin{array}{c}\text { Flux } \\
\left(10^{-13} \mathrm{erg} \mathrm{cm}^{-2} \mathrm{~s}^{-1}\right)\end{array}$ & $\alpha_{X}$ & $\theta_{\text {jet }}$ & Ref. \\
\hline 000528 & $\ldots$ & 11 & $2.3 \pm 1.0$ & $\ldots$ & $\ldots$ & 12 \\
\hline 000529 & $\ldots$ & 9.0 & 2.8 & $\ldots$ & $\ldots$ & 26 \\
\hline \multirow[t]{2}{*}{000926} & 2.037 & 54.9 & $2.23 \pm 0.77$ & $-3.7 \pm 1.5^{a}$ & 0.140 & 14,27 \\
\hline & & 66.5 & $0.94 \pm 0.14$ & & & 27 \\
\hline 001025 & $\ldots$ & 50.4 & $0.53 \pm 0.10$ & $-3 \pm 1.9^{a}$ & $\ldots$ & 28 \\
\hline 001109 & $\ldots$ & 19.3 & $7.1 \pm 0.5$ & $\ldots$ & $\ldots$ & 29 \\
\hline \multirow[t]{2}{*}{010214} & $\ldots$ & 7.7 & 6 & $<-1.6$ & $\ldots$ & 30 \\
\hline & & 24.1 & $<0.5$ & & & 30 \\
\hline 010220 & $\ldots$ & 20.8 & 0.33 & $-1.2 \pm 1.0$ & $\ldots$ & 28 \\
\hline \multirow[t]{3}{*}{010222} & 1.477 & 8.9 & $101 \pm 11$ & $-1.33 \pm 0.04$ & 0.080 & 14,31 \\
\hline & & 32.7 & $18.7 \pm 1.8$ & & & 31 \\
\hline & & 54.4 & $9.9 \pm 0.5$ & & & 31 \\
\hline 011211 & 2.14 & 11.0 & 1.9 & $-1.7 \pm 0.2$ & $\ldots$ & 32 \\
\hline 020322 & $\ldots$ & 18.8 & $3.5 \pm 0.2$ & $-1.26 \pm 0.23$ & $\ldots$ & 33 \\
\hline 020405 & 0.698 & 41.0 & $13.6 \pm 2.5$ & $-1.15 \pm 0.95^{d}$ & 0.285 & $34,35,36$ \\
\hline 020813 & 1.254 & 31.9 & 22 & $-1.42 \pm 0.05$ & 0.066 & 37,38 \\
\hline 021004 & 2.323 & 31.4 & $4.3 \pm 0.7$ & $-1.0 \pm 0.2$ & 0.240 & 39,40 \\
\hline
\end{tabular}

Note. - The columns are (left to right): (1) GRB name, (2) redshift, (3) midpoint epoch of X-ray observation, (4) X-ray flux, (5) temporal decay index $\left(F_{X} \propto\right.$ $\left.t^{\alpha_{X}}\right)$, (6) jet opening angle, and (7) references for the X-ray flux and jet opening angle. ${ }^{a}$ Due to the large uncertainty in the value of $\alpha_{X}$ we use the median value for the sample, $\left\langle\alpha_{X}\right\rangle=-1.33 \pm 0.38 .{ }^{b}$ The redshift is based on matching the optical lightcurve of SN 1998bw to the red excess reported by Bloom et al. (1999). ${ }^{c}$ The redshift limit is based on a detection of the afterglow in the optical $U$-band (Jaunsen et al. 2001). ${ }^{d}$ The inferred jet break is at $t=0.95$, prior to the X-ray observation - we use the model fit to extrapolate the flux to $t=10 \mathrm{hr}$ (Berger et al. in prep.)

References. - (1) Feroci et al. (1998); (2) Piro (2001); (3) Frontera et al. (1998); (4) Piro et al. (1998); (5) Frail et al. (2001); (6) Murakami et al. (1997); (7) Smith et al. (2002); (8) Antonelli et al. (1999); (9) Marshall \& Takeshima (1998); (10) in 't Zand et al. (1998); (11) Yost et al. (2002); (12) De Pasquale et al. (2002); (13) Nicastro et al. (1999); (14) Panaitescu \& Kumar (2002); (15) Vreeswijk et al. (1999); (16) Frontera et al. (2000); (17) Pian et al. (2001); (18) Nicastro et al. (1999); (19) Feroci et al. (2001); (20) Amati et al. (2000); (21) Frontera et al. (1999): (22) Takeshima et al. (1999); (23) Marshall et al. (2000): (24) Piro et 
Table 2. X-ray Afterglow Data at $t=10 \mathrm{hr}$

\begin{tabular}{|c|c|c|c|c|c|}
\hline GRB & $z$ & $\begin{array}{c}F_{X, 10} \\
\left(10^{-13} \mathrm{erg} \mathrm{cm}^{-2} \mathrm{~s}^{-1}\right)\end{array}$ & $\begin{array}{c}L_{X, i s o} \\
\left(10^{45} \mathrm{erg} \mathrm{s}^{-1}\right)\end{array}$ & $\theta_{\text {jet }}$ & $\begin{array}{c}L_{X} \\
\left(10^{44} \mathrm{erg} \mathrm{s}^{-1}\right)\end{array}$ \\
\hline 970111 & $\ldots$ & $3.36 \pm 1.64$ & $2.56 \pm 1.25$ & $\cdots$ & $\ldots$ \\
\hline 970228 & 0.695 & $27.50 \pm 3.17$ & $6.82 \pm 0.79$ & $\cdots$ & $\cdots$ \\
\hline 970402 & $\ldots$ & $2.86 \pm 0.61$ & $2.18 \pm 0.46$ & $\ldots$ & $\cdots$ \\
\hline 970508 & 0.835 & $9.60 \pm 1.47$ & $3.74 \pm 0.57$ & 0.391 & $2.82 \pm 0.43$ \\
\hline 970815 & $\cdots$ & $<18.47$ & $<14.1$ & $\ldots$ & . \\
\hline 970828 & 0.958 & $32.12 \pm 6.31$ & $17.6 \pm 3.4$ & 0.128 & $1.44 \pm 0.28$ \\
\hline 971214 & 3.418 & $7.29 \pm 0.87$ & $89.6 \pm 10.8$ & $>0.100$ & $>4.48$ \\
\hline 971227 & $\cdots$ & $4.38 \pm 1.26$ & $3.34 \pm 0.96$ & $\cdots$ & $\cdots$ \\
\hline 980326 & $\sim 1$ & $<12.89$ & $<9.82$ & $<0.110$ & $<0.59$ \\
\hline 980329 & $\cdots$ & $10.68 \pm 2.10$ & $8.14 \pm 1.60$ & 0.081 & $0.27 \pm 0.05$ \\
\hline 980515 & $\cdots$ & $2.27 \pm 0.90$ & $1.73 \pm 0.69$ & $\cdots$ & $\cdots$ \\
\hline 980519 & $\cdots$ & $6.14 \pm 1.89$ & $4.68 \pm 1.44$ & 0.040 & $0.04 \pm 0.01$ \\
\hline 980613 & 1.096 & $7.03 \pm 2.28$ & $5.36 \pm 1.74$ & $>0.226$ & $>1.36$ \\
\hline 980703 & 0.966 & $18.24 \pm 4.97$ & $10.2 \pm 2.8$ & 0.200 & $2.03 \pm 0.55$ \\
\hline 981226 & $\ldots$ & $6.19 \pm 1.20$ & $4.72 \pm 0.92$ & $\ldots$ & $\cdots$ \\
\hline 990123 & 1.600 & $66.09 \pm 6.33$ & $128.31 \pm 12.29$ & 0.089 & $5.08 \pm 0.49$ \\
\hline 990217 & $\cdots$ & $<1.25$ & $<0.95$ & $\cdots$ & $\cdots$ \\
\hline 990510 & 1.619 & $41.07 \pm 3.68$ & $82.09 \pm 7.35$ & 0.054 & $1.20 \pm 0.11$ \\
\hline 990627 & $\cdots$ & $4.41 \pm 0.85$ & $3.36 \pm 0.65$ & $\cdots$ & $\cdots$ \\
\hline 990704 & $\cdots$ & $10.23 \pm 3.34$ & $7.80 \pm 2.54$ & $\cdots$ & $\cdots$ \\
\hline 990705 & 0.840 & $3.11 \pm 1.14$ & $1.23 \pm 0.45$ & 0.096 & $0.06 \pm 0.02$ \\
\hline 990806 & $\cdots$ & $8.46 \pm 3.14$ & $6.45 \pm 2.39$ & $\cdots$ & $\cdots$ \\
\hline 990907 & $\cdots$ & $11.58 \pm 6.95$ & $8.82 \pm 5.29$ & $\cdots$ & $\cdots$ \\
\hline 991014 & $\ldots$ & $4.54 \pm 1.71$ & $3.46 \pm 1.30$ & $\ldots$ & $\cdots$ \\
\hline 991216 & 1.020 & $287.21 \pm 14.73$ & $183.22 \pm 9.39$ & 0.051 & $2.38 \pm 0.12$ \\
\hline 000115 & $\cdots$ & $78.3 \pm 14.12$ & $59.67 \pm 10.76$ & $\cdots$ & $\cdots$ \\
\hline 000210 & 0.846 & $4.56 \pm 1.16$ & $1.83 \pm 0.47$ & $\cdots$ & $\cdots$ \\
\hline 000214 & $\cdots$ & $10.25 \pm 2.16$ & $7.81 \pm 1.65$ & $\cdots$ & $\cdots$ \\
\hline 000528 & $\cdots$ & $2.61 \pm 1.27$ & $1.99 \pm 0.97$ & $\cdots$ & $\cdots$ \\
\hline 000529 & $\ldots$ & $2.43 \pm 0.47$ & $1.85 \pm 0.36$ & $\cdots$ & $\cdots$ \\
\hline
\end{tabular}


Table 2-Continued

\begin{tabular}{cccccc}
\hline \hline GRB & $z$ & $\begin{array}{c}F_{X, 10} \\
\left(10^{-13} \mathrm{erg} \mathrm{cm}^{-2} \mathrm{~s}^{-1}\right)\end{array}$ & $\begin{array}{c}L_{X, \text { iso }} \\
\left(10^{45} \mathrm{erg} \mathrm{s}^{-1}\right)\end{array}$ & $\begin{array}{c}\theta_{\text {jet }} \\
\left(10^{44} \mathrm{erg} \mathrm{s}^{-1}\right)\end{array}$ \\
\hline 000926 & 2.037 & $20.41 \pm 8.06$ & $71.69 \pm 28.31$ & 0.140 & $7.01 \pm 2.77$ \\
001025 & $\ldots$ & $67.85 \pm 51.48$ & $51.71 \pm 39.22$ & $\ldots$ & $\ldots$ \\
001109 & $\ldots$ & $17.02 \pm 2.06$ & $12.97 \pm 1.57$ & $\ldots$ & $\ldots$ \\
010214 & $\ldots$ & $3.95 \pm 0.80$ & $3.01 \pm 0.61$ & $\ldots$ & $\ldots$ \\
010220 & $\ldots$ & $0.79 \pm 0.21$ & $0.61 \pm 0.16$ & $\ldots$ & $\ldots$ \\
010222 & 1.477 & $86.50 \pm 9.88$ & $137.86 \pm 15.75$ & 0.080 & $4.41 \pm 0.50$ \\
011211 & 2.14 & $2.23 \pm 0.39$ & $8.86 \pm 1.56$ & $\ldots$ & $\ldots$ \\
020322 & $\ldots$ & $7.75 \pm 0.67$ & $5.91 \pm 0.51$ & $\ldots$ & $\ldots$ \\
020405 & 0.698 & $68.98 \pm 20.21$ & $17.29 \pm 5.07$ & 0.285 & $6.98 \pm 2.04$ \\
020813 & 1.254 & $113.98 \pm 17.01$ & $121.21 \pm 18.09$ & 0.066 & $2.61 \pm 0.39$ \\
021004 & 2.323 & $13.50 \pm 2.47$ & $65.36 \pm 11.95$ & 0.240 & $18.7 \pm 3.4$ \\
\hline
\end{tabular}

Note. - The columns are (left to right): (1) GRB name, (2) redshift, (3) $\mathrm{X}$-ray flux at $t=10 \mathrm{hr}$, (4) X-ray luminosity at $t=10 \mathrm{hr}$, (5) jet opening angle, and (6) beaming-corrected X-ray luminosity at $t=10 \mathrm{hr}$. 

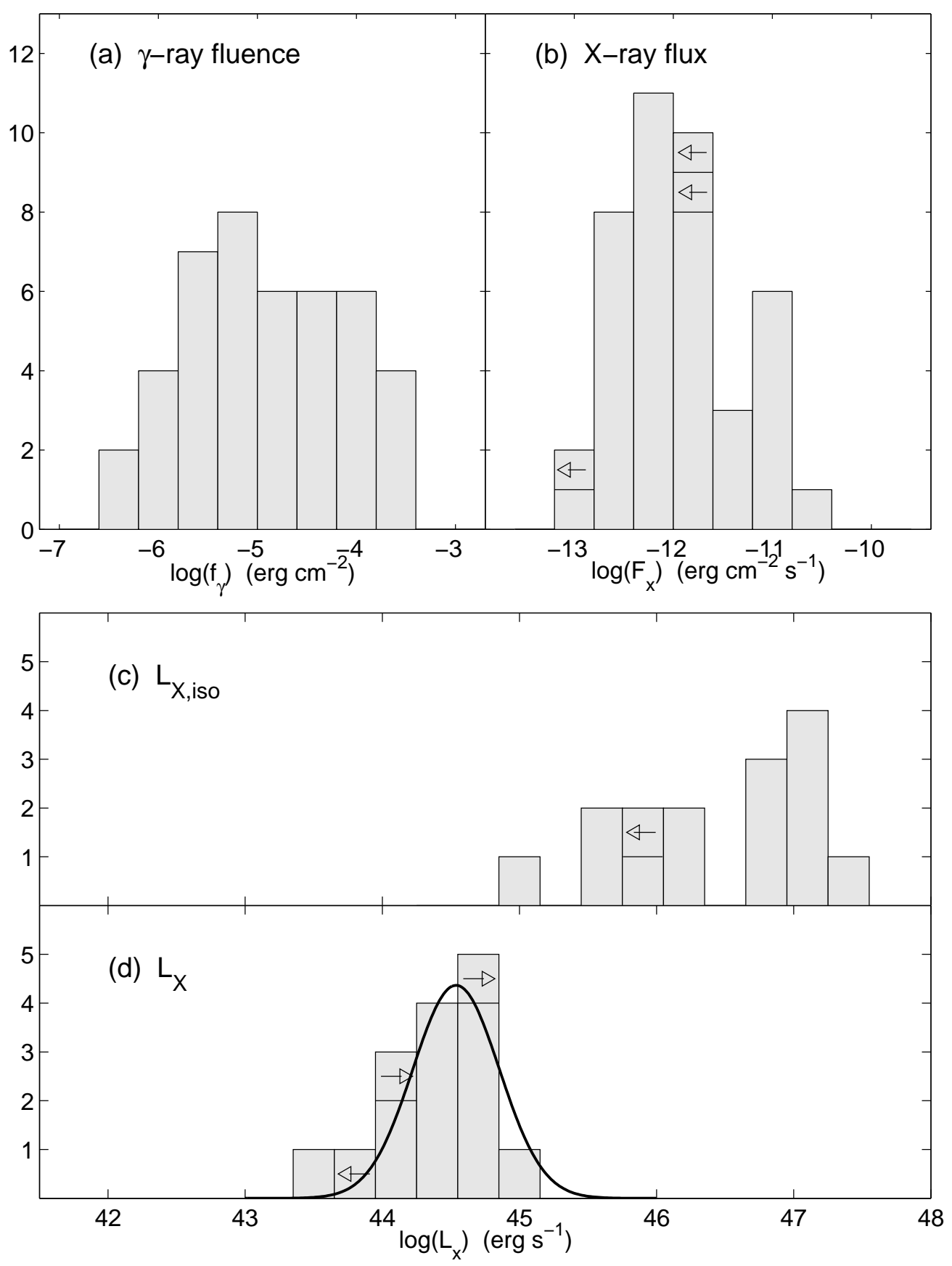

Fig. 1.- Panel (a) shows the distribution of $\gamma$-ray fluences. Panel (b) shows the distribution of X-ray fluxes scaled to $t=10 \mathrm{hr}$ after the burst. In panel (c) we plot the isotropic-equivalent $\mathrm{X}$-ray luminosity, $L_{X \text {,iso }}$, for the subset of X-ray afterglows with known $\theta_{j}$ and redshift, while in panel (d) we show the true X-ray luminosity, $L_{X}=f_{b}^{-1} L_{X \text {,iso. }}$. 


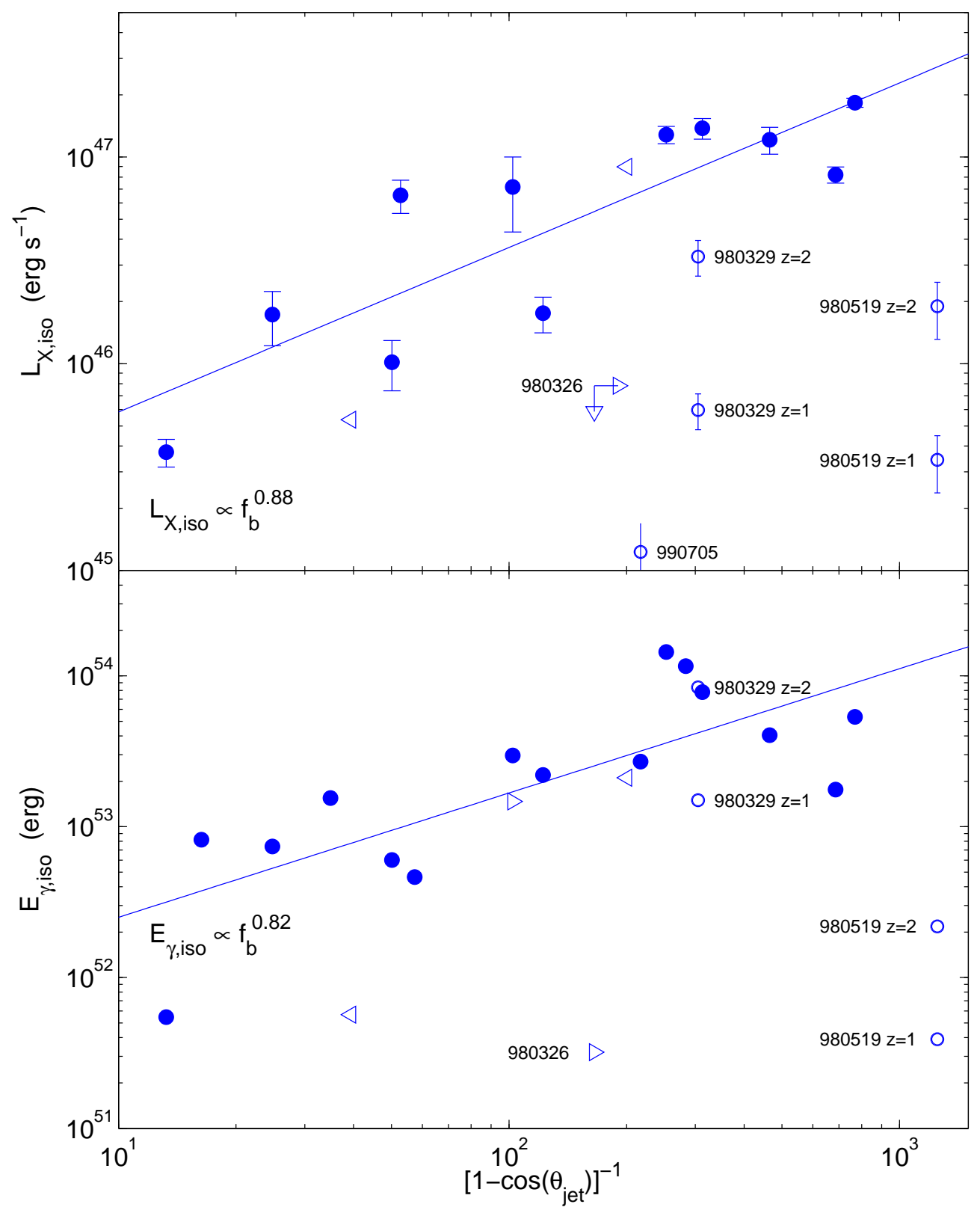

Fig. 2.- Isotropic-equivalent X-ray luminosity (top) and isotropic-equivalent $\gamma$-ray energy (bottom) as a function of the beaming factor, $\left[1-\cos \left(\theta_{j}\right)\right]^{-1}$. There is a strong positive correlation between $L_{X \text {,iso }}$ and $f_{b}^{-1}$, as well as between $E_{\gamma \text {,iso }}$ and $f_{b}^{-1}$ resulting in an approximately constant true $\mathrm{X}$-ray luminosity and $\gamma$-ray energy release. In fact, while the distributions of all three parameters span about three orders of magnitude, the distributions of the beaming-corrected parameters span about one order of magnitude. 